\title{
L'évolution au péril des médias : néocréationnisme et dessein intelligent !
}

C'est souvent avec une certaine condescendance que sont accueillis en France les échos des procès intentés aux États-Unis contre ceux qui voudraient introduire le créationnisme dans les programmes scolaires au même titre que le darwinisme. Une telle prétention, pensons-nous, ne peut voir le jour que là où les fondamentalistes chrétiens sont puissants en nombre et en moyens financiers. Mais, il faut se rendre à l'évidence, la vague intégriste a atteint l'Europe et ses effets commencent à se faire sentir en France sous prétexte de recherches sur les relations entre la science et la religion.

Le plus dangereux est que l'opposition au darwinisme avance masquée par le biais de l'intelligent design, ou "dessein intelligent », forme moderne du créationnisme pour ceux qui acceptent l'évolution mais ne peuvent se résoudre à ce que l'homme n'en soit que le produit accidentel. Pour eux, notre présence sur terre a été programmée par un «dessein divin » et ils considèrent que nous en sommes l'actuel aboutissement.

Les articles sur ce sujet se multiplient dans la presse "grand public », et la télévision s'en mêle maintenant avec plusieurs documentaires présentés à des heures de grande écoute, tel le Dieu contre Darwin d'Arte en septembre 2006.

La principale faiblesse des propos tenus dans ces documentaires est que le mécanisme de sélection naturelle n'y est jamais évoqué. Or, affirmer que l'adaptation au milieu n'est pas le moteur principal de l'évolution n'a pas de sens si on ne précise pas, au préalable, que c'est à travers ce mécanisme que se fait cette adaptation et qu'elle peut s'étendre sur plusieurs générations : l'environnement ne crée rien, il ne fait que sélectionner. Le changement climatique étant le grand problème du moment, on explique souvent, à propos de telle ou telle espèce animale, que, le milieu dans lequel elle vivait ayant changé, elle a dû modifier son mode de vie, en particulier son régime alimentaire, entraînant ainsi une transformation de l'espèce en question. Mais on ne précise jamais que cette transformation se fait sur plusieurs générations par le jeu de la sélection naturelle.

L'absence d'oppositions manifestes au darwinisme en France entraîne sans doute que les chercheurs, dans leurs discours ou dans leurs communications avec le public, ne prennent pas assez de précautions pour séparer ce qui est scientifiquement démontré, à savoir que les êtres vivants ont évolué dès l'apparition de la vie sur terre, de ce qui est encore à l'état d'hypothèses, à savoir les théories sur les mécanismes de cette évolution. Certes, on en sait davantage à ce sujet depuis l'époque de Darwin, et plus particulièrement depuis la découverte des supports de l'hérédité que sont les gènes et l'ADN qu'ils contiennent. Mais, au fur et à mesure des recherches, ce qui paraissait assuré dans les années 1970 ou 1980 l'est beaucoup moins aujourd'hui, avec la mise en évidence que l'ADN n'est pas l'unique porteur de l'information génétique et avec l'ouverture aux chercheurs du vaste champ de l'épigénétique. Quoi qu'il en soit, quand apparaissent des formes nouvelles au hasard des mutations, c'est toujours à travers le processus de sélection naturelle que se fait le tri. Celles qui possèdent des avantages adaptatifs sont destinées à se perpétuer, tandis que celles qui n'en ont pas sont amenées à disparaître immédiatement ou au bout de quelques générations.

Cependant, la percée actuelle en France de l'intelligent design oblige à plus de vigilance. Après les documentaires sur Arte, c'est au tour d'un grand quotidien comme Le Monde de s'emparer du sujet en proposant une double page sur le "néocréationnisme français » (Le Monde, 2 septembre 2006).

Il est vrai que la communauté scientifique n'a sans doute pas été assez attentive aux dérives imposées à la théorie darwinienne de l'évolution, lors d'extrapolations dans des domaines qui ne la concernaient pas. Songeons en particulier au darwinisme social - qui veut appliquer aux hommes le principe de sélection naturelle pour justifier l'élimination des plus faibles - et à la notion 
de progrès dans le domaine culturel. On peut, en effet, considérer que cette notion de progrès est en phase avec les tenants de l'intelligent design pour lesquels l'apparition de l'homme, avec des capacités spirituelles supérieures à celles des autres êtres vivants, est le but ultime de l'évolution. Par contre, elle peut heurter les créationnistes pour qui, conformément au récit biblique, l'homme est créé par Dieu à son image en même temps que les autres créatures, et pour lesquels l'aboutissement final est l'Apocalypse et le Jugement dernier. Pour les créationnistes, la croyance en un début du monde est corrélée à celle d'une fin. Toute leur vie est gouvernée par cette croyance et donc par ce qui est à la fois une espérance et un objet de terreur, la Résurrection. Face à de telles convictions, il est inutile d'avancer des arguments scientifiques quels qu'il soient.

Il ne s'agit pas d'empêcher certains de croire que l'existence du monde est l'œuvre d'un créateur divin, mais d'exiger que les théories incluant l'existence de ce dernier s'affirment ouvertement comme telles et n'avancent pas masquées, ce qui est souvent le cas de l'intelligent design, qui prétend s'appuyer sur des preuves scientifiques. Et c'est bien à la communauté scientifique de savoir se montrer vigilante et de contribuer à débusquer les faux arguments, afin de ne pas permettre aux théories basées sur ceux-ci de s'infiltrer subrepticement dans l'esprit des citoyens.

Mais c'est aussi à la communauté scientifique de s'interroger sur la signification de ces questionnements, de leur succès auprès de certains cercles, et donc de s'interroger sur elle-même en mettant à l'épreuve ses propres certitudes. NSS ouvrira dans l'année un dossier de réflexion permettant à cette communauté d'exprimer dans sa diversité des points de vue de scientifiques sur la question complexe de l'évolution et, au-delà, sur les multiples formes de la relation entre sciences et raison.

Claudine Friedberg

To access this journal online: www.edpsciences.org 